Research Paper

\title{
Comparison of the RECIST 1.0 and RECIST 1.1 in Non-Small Cell Lung Cancer Treated with Cytotoxic Chemotherapy
}

\author{
Hyun Chang Choi ${ }^{*}$, Jung Han Kim ${ }^{1 * 凶}$, Hyeong Su Kim , Soong Goo Jung1, Sang Muk Hwang1, Sung Bae \\ $\mathrm{Ju}^{1}$, Ik Yang ${ }^{2}$ \\ 1. Department of Internal Medicine, Kangnam Sacred Heart Hospital, Hallym University Medical Center, Hallym University College of \\ Medicine, Seoul 150-950, Republic of Korea \\ 2. Department of Radiology, Kangnam Sacred Heart Hospital, Hallym University Medical Center, Hallym University College of Medicine, \\ Seoul 150-950, Republic of Korea \\ * Hyun Chang Choi and Jung Han Kim equally contributed to this study.
}

$\triangle$ Corresponding author: Jung Han Kim, MD. PhD., Department of Internal Medicine, Kangnam Sacred-Heart Hospital, Hallym University Medical Center, Shingil-ro1, Youngdeungpo-Gu, Seoul 150-950, South Korea. E-mail: harricil@hotmail.com, harricil@hallym.or.kr

(C) 2015 Ivyspring International Publisher. Reproduction is permitted for personal, noncommercial use, provided that the article is in whole, unmodified, and properly cited. See http://ivyspring.com/terms for terms and conditions.

Received: 2015.02.05; Accepted: 2015.05.05; Published: 2015.05.27

\begin{abstract}
Background : The impact of the RECIST 1.1 on the selection of target lesions and assessment of tumor response was not evaluated in patients with advanced NSCLC who received cytotoxic chemotherapy.

Methods: We reviewed medical records of patients with advanced NSCLC who received first-line chemotherapy between January 2004 and December 2013 and compared the selection of target lesions and tumor responses using the two RECIST versions.

Results: A total of 88 patients who had at least one target lesion according to the RECIST 1.0 were included in the study. The number of target lesions by the RECIST 1.1 was significantly lower than that by the RECIST 1.0. When adopting the RECIST 1.1 instead of the RECIST 1.0, 40 patients (45.4\%) showed a decrease in the number of target lesions. Three patients no longer had target lesion because of the new lymph node (LN) criteria of the RECIST 1.1. Tumor responses showed a high level of concordance between the RECIST 1.0 and RECIST 1.1, with a kappa value of 0.912 . Four patients (4.5\%) showed disagreement of tumor responses between the two criteria, which were all due to the change of the LN criteria.

Conclusion: The RECIST 1.1 showed a high level of concordance with the RECIST 1.0 in the assessment of tumor response in advanced NSCLC patients treated with cytotoxic chemotherapy. The new LN criteria were the major cause of the reduction of target lesions and reclassification of the tumor response.
\end{abstract}

Key words: RECIST 1.0; RECIST 1.1; Lung cancer; Chemotherapy; Target lesion

\section{Introduction}

Researchers usually rely on the World Health Organization (WHO) guidelines or the Response Evaluation Criteria in Solid Tumors (RECIST) criteria to define tumor response. The WHO guidelines have been used as the standard method for assessing tumor response since the early 1980s [1]. Tumor burden is estimated by summing the products of bi-dimensional measurements. Because the criteria for selecting and measuring target lesions were not clearly described in the WHO guidelines, however, tumor response as- 
sessment has not been accurately reproducible between investigators [2-4]. The RECIST Working Group proposed the RECIST guideline version 1.0 (RECIST 1.0) as the new response criteria in 2000 [5]. The RECIST 1.0 defined the minimum size of measurable lesion by computed tomography (CT) and incorporated uni-dimensional measurement instead of the two-dimensional method in the WHO guidelines. The RECIST 1.0 criteria also adopted a total of 10 target lesions with a maximum of 5 lesions per organ. It has been widely accepted as the standardized criteria for tumor response assessment, quickly replacing the WHO guidelines. However, a number of questions and issues including the number of target lesions and the size of lymph nodes (LNs) to be assessed were raised on the RECIST 1.0. In addition, the rapid innovations of new imaging technologies, such as multi-detector computed tomography (MDCT) and positron emission tomography combined with $\mathrm{CT}$ (PET/CT), have necessitated the revision of the guidelines [6].

Based on the database of about 6,500 patients from 16 clinical trials, the RECIST Working Group published the revised version of the RECIST guidelines (RECIST 1.1) in 2009 [7-11]. Major changes in the RECIST 1.1 involved the reduction of target lesions, the inclusion of bone lesions as possible target lesions, the criteria for assessing pathologic LNs, and the clarification of progressive disease (PD) and unequivocal progression of non-target lesions [12]. The maximum number of target lesions to be assessed is reduced from ten to five in total, and from five to two per organ. Lytic or mixed lytic-blastic bone lesion with measurable soft tissue component is newly regarded as a target lesion according to the RECIST 1.1. The new LN criteria recommend the short-axis measurement and only consider LNs of at least $15 \mathrm{~mm}$ target lesions. LNs with at least $10 \mathrm{~mm}$ but less than $15 \mathrm{~mm}$ along short axis, even though which may be pathological, are regarded as non-target lesions, and LNs with a short axis of less than $10 \mathrm{~mm}$ are defined as nonpathologic. In addition to the at least $20 \%$ increase in the tumor measurement, PD according to the RECIST 1.1 requires an absolute increase of at least 5 $\mathrm{mm}$ of the sum of the longest diameters of target lesions. The RECIST 1.1 also recommends incorporating $\mathrm{PET} / \mathrm{CT}$ in the detection of new lesions.

The RECIST 1.1 has shown almost perfect agreement with the RECIST 1.0 in the assessment of tumor response in patients with advanced gastric cancer (GC) $[13,14]$, metastatic colorectal cancer (CRC) [15], and thyroid cancer [16] . In patients with advanced non-small cell lung cancer (NSCLC) who received epidermal growth factor tyrosine kinase inhibitors (EGFR-TKIs), the RECIST 1.1 also revealed high concordance with the RECIST 1.0 [17-19]. To the best of our knowledge, however, the impact of the RECIST 1.1 on the selection of target lesions and assessment of tumor response was not investigated in patients with advanced NSCLC who received cytotoxic chemotherapy.

We conducted this study to compare CT tumor measurement and tumor response assessment between the RECIST 1.0 and the RECIST 1.1 in advanced NSCLC patients who were treated with cytotoxic chemotherapy.

\section{Patients and methods}

\section{Patients}

This study obtained Institutional Review Board's approval with a waiver of patient's informed consent according to the Korean Ethical Guidelines for epidemiological research. We reviewed medical records of patients with advanced NSCLC who received cytotoxic chemotherapy as first-line treatment between January 2004 and December 2013 at Kangnam Sacred Heart Hospital, Seoul, South Korea. The patients were eligible if they had following criteria; histologically confirmed non-small cell carcinoma of the lung, radiologically or histologically confirmed advanced disease (stage IIIB or IV), at least one measurable lesion by the RECIST 1.0, no history of other cancer, no history of previous chemotherapy or radiotherapy, and CT examinations at baseline and after chemotherapy. Patients who had shown unequivocal progression of non-target lesions or development of new lesions at the follow-up CT were excluded from the final analyses because they would have the same response classification between the two criteria.

\section{CT examinations}

Chest CT scans for evaluating tumor response were obtained at baseline and after 2 or 3 cycles of first-line chemotherapy. All scans were obtained with the MDCT scanner (SOMATOM Sensation, Giemens Healthcare, Forchheim, Germany) with intravenous administration of contrast medium, iopromide (Ultravist 300, Bayer Medical Systems), with a scan delay of 20-30 seconds. Patients were scanned from the clavicles to the adrenal glands at end-inspiration in supine position. Axial images were reconstructed with a slice thickness of $5 \mathrm{~mm}$ and were transferred to the Picture Archiving and Communication System (PACS) workstation (PiView Star, INFINITT Healthcare Co. LTD., Seoul, South Korea).

\section{Tumor measurements}

We evaluated each patient's tumor measurements from the CT scans and assessed tumor responses with no interval confirmation. Tumor meas- 
urements were performed manually on the axial CT image planes using calipers of a measuring tool on the PACS. The measurement of target lesions and sum of the longest diameters, the description of non-target lesions, the development of new lesions, and the tumor response for each patient were recorded by the consensus of two experienced investigators according to the RECIST 1.0 and RECIST 1.1, respectively. For cases showing a significant discrepancy between the two investigators, a board-certified chest radiologist re-evaluated the CT images.

\section{Statistical analyses}

A paired Student's $t$ test was used to estimate the statistical significance of changes in the number of target lesions at baseline between the RECIST 1.0 and RECIST 1.1. The $\chi^{2}$ test was used to compare the proportion of patients and the overall response rate (ORR) between two groups. All P values were based on a two-sided hypothesis, with a value of less than 0.05 being considered significant. The concordance level of tumor responses between the two criteria was assessed using карpa statistics. A kappa value of more than 0.75 was interpreted as showing excellent agreement.

\section{Results}

\section{Patient characteristics}

During the study period, a total of 129 patients with advanced NSCLC received first-line chemotherapy with a variety of cytotoxic regimens. Fifteen patients $(11.6 \%)$ had not been evaluated for tumor response, and nine $(7 \%)$ had no target lesion according to the RECIST 1.0. Six patients (4.6\%) received first-line treatment with an EGF R-TKI. According to the inclusion criteria, eleven patients $(8.5 \%)$ who showed the substantial progression of non-target lesion or development of new lesions were also excluded from the study. Finally, a total of 88 patients $(68.2 \%)$ who had at least one measurable lesion in any one organ were included in the final analyses.

Patients' baseline characteristics are summarized in Table 1. The patients consisted of 65 male $(73.9 \%)$ and 23 female, with a median age of 61 years (range, 29-89 years). Forty-three patients (48.9\%) had adenocarcinoma and thirty-two $(36.4 \%)$ had squamous cell carcinoma. Eighty-one $(92.0 \%)$ patients had a stage IV NSCLC, and the remaining seven had a stage IIIB disease. Almost all patients (95.5\%) had at least one measurable lesion in the lungs. The most common metastatic site with measurable target lesions was the LNs (47.8\%), followed by the liver (13.6\%). According to the RECIST 1.1, three patients newly had target lesions in the bones. Twenty patients $(22.7 \%)$ had a single target lesion according to the RECIST 1.0.
Thirty-seven patients $(42.0 \%)$ had target lesions in two organs, most commonly in the lungs and LNs. Seven patients $(7.9 \%)$ had target lesions in 3 or more organs.

Forty patients $(45.5 \%)$ received taxane (paclitaxel, docetaxel, or genexol PM) with or without platinum (cisplatin or carboplatin), and $22(25.0 \%)$ were treated with gemcitabine with or without platinum.

Table 1. Characteristics of the 88 patients.

\begin{tabular}{lll}
\hline Characteristics & No. of patients & $\%$ \\
\hline $\begin{array}{l}\text { Median age, years (range) } \\
\text { Gender }\end{array}$ & $61(29-89)$ & \\
Male & 65 & 73.9 \\
Female & 23 & 26.1 \\
Stage & & \\
IIIB & 7 & 8.0 \\
IV & 81 & 92.0 \\
Histology & & \\
Adenocarcinoma & 43 & 48.9 \\
Squamous cell carcinoma & 32 & 36.4 \\
Large cell carcinoma & 3 & 3.4 \\
Unclassifiable & 10 & 11.4 \\
Measurable target lesions & & \\
Lungs & 84 & 95.5 \\
Lymph nodes & 42 & 47.8 \\
Liver & 12 & 13.6 \\
Adrenal glands & 4 & 4.5 \\
Bones & 3 & 3.4 \\
Pancreas & 1 & 1.1 \\
First-line chemotherapy regimens & & \\
Taxane +/- platinum & 40 & 45.5 \\
Gemcitabine +/- platinum & 22 & 25.0 \\
Irinotecan +/- platinum & 12 & 13.6 \\
Pemetrexed +/- platinum & 9 & 10.2 \\
Etoposide +/- platinum & 5 & 5.9 \\
\hline
\end{tabular}

\section{Number of target lesions}

The number of target lesions according to the RECIST 1.1 was significantly lower than that according to RECIST $1.1(\mathrm{P}<0.001)$. The median number of target lesions was 4 (range, 1-9) by the RECIST 1.0 and 3 (range, 1-5) by the RECIST 1.1, respectively. Forty patients $(45.4 \%)$ showed a decrease in the number of target lesions by adopting the RECIST 1.1. Two patients who had a $\mathrm{LN} \leq 15 \mathrm{~mm}$ along short axis showed no change in the number of target lesions due to the inclusion of bone lesion with a soft tissue component according to the RECIST 1.1. In one patient, the number of target lesions was increased because of bone target lesion newly defined according to the RECIST 1.1.

The reduction of the maximum number of target lesions (from 10 to 5 in total, and from 5 to 2 per organ) resulted in the decrease of target lesions in 3 patients. Ten patients showed a decrease of target lesions because of both the new LN criteria and the reduced maximum number of target lesions. In the re- 
maining 27 patients, the decrease was due to the new LN criteria ( $\geq 15 \mathrm{~mm}$ along the short axis to be considered pathological). Out of these 27 patients, three no longer had target lesions when adopting the RECIST 1.1 because all their target lesions were LNs less than $15 \mathrm{~mm}$ along short axis. Among 123 patients who received first-line chemotherapy during the study period, the proportion of patients with no measurable lesion was increased from $7.3 \%(9 / 123)$ by the RECIST 1.0 to $9.75 \%(12 / 123)$ by the RECIST 1.1 (P $=0.494)$.

\section{Tumor responses}

The percentage changes in the sum of tumor measurements respectively according to the RECIST 1.0 and RECIST 1.1 are presented in Figure 1. Thirty-three patients $(38.8 \%)$ showed an increase (range, $1.4-21.2 \%)$ in the absolute value of the percentage change when adopting the RECIST 1.1, instead of the RECIST 1.0. Fifteen patients $(17.6 \%)$ showed a reduction in the change rate (range, $0.5-10.5 \%$ ) of the sum of tumor measurements.

The comparison of tumor responses between the two criteria is shown in Table 2. The tumor responses showed a high level of concordance between the RECIST 1.0 and RECIST 1.1, with a kappa value of 0.912 (95\% confidence interval, 0.827-0.996). Only four patients $(4.5 \%)$ showed disagreement of the tumor responses between the two criteria. One patient with partial response (PR) according to the RECIST 1.0 was reclassified as complete response $(\mathrm{CR})$ because $\mathrm{LN}$ with short axis $\leq 10 \mathrm{~mm}$ was considered normal according to the RECIST 1.1. Two patients defined as stable disease (SD) by the RECIST 1.0 were reclassified as PR by the RECIST 1.1 because of the decreased LN target lesions. The remaining one patient had PD according to the RECIST 1.0 but was re-categorized as SD according to the RECIST 1.1 because a new LN measuring $\leq 10 \mathrm{~mm}$ along short axis did not meet the pathologic LN criteria of the RECIST 1.1. The ORR of first-line chemotherapy was not significantly different between the two criteria $(27.1 \%$ according to the RECIST 1.0 vs. $29.4 \%$ according to the RECIST $1.1, \mathrm{P}=$ $0.733)$.

\section{Discussion}

In the current study, we compared tumor measurement and response assessment by CT based on the RECIST 1.0 and RECIST 1.1 in patients with advanced NSCLC who had received cytotoxic chemotherapy as first-line treatment. Our data showed that the RECIST 1.1 significantly reduced the number of target lesions to be measured for assessing tumor response. However, there was almost perfect agreement in the assessment of tumor responses between the RECIST 1.0 and RECIST 1.1.

Table 2. Comparison of tumor responses by the RECIST 1.0 versus RECIST 1.1.

\begin{tabular}{llllll}
\hline \multirow{2}{*}{$\begin{array}{l}\text { Tumor response } \\
\text { by RECIST } 1.0\end{array}$} & \multicolumn{4}{l}{ Tumor response by RECIST 1.1 } & Total \\
\cline { 2 - 5 } & CR & PR & SD & PD & 0 \\
CR & 0 & 0 & 0 & 0 & 23 \\
PR & 1 & 22 & 0 & 0 & 53 \\
SD & 0 & 2 & 51 & 0 & 9 \\
PD & 0 & 0 & 1 & 8 & 85 \\
Total & 1 & 24 & 52 & 8 &
\end{tabular}

The overall response rates were $27.1 \%$ by the RECIST 1.0 and $29.4 \%$ by the RECIST $1.1(\mathrm{P}=0.733)$

The level of concordance of the tumor responses between the two criteria is 0.912 .

\section{RECIST $1.0 \quad$ RECIST 1.1}

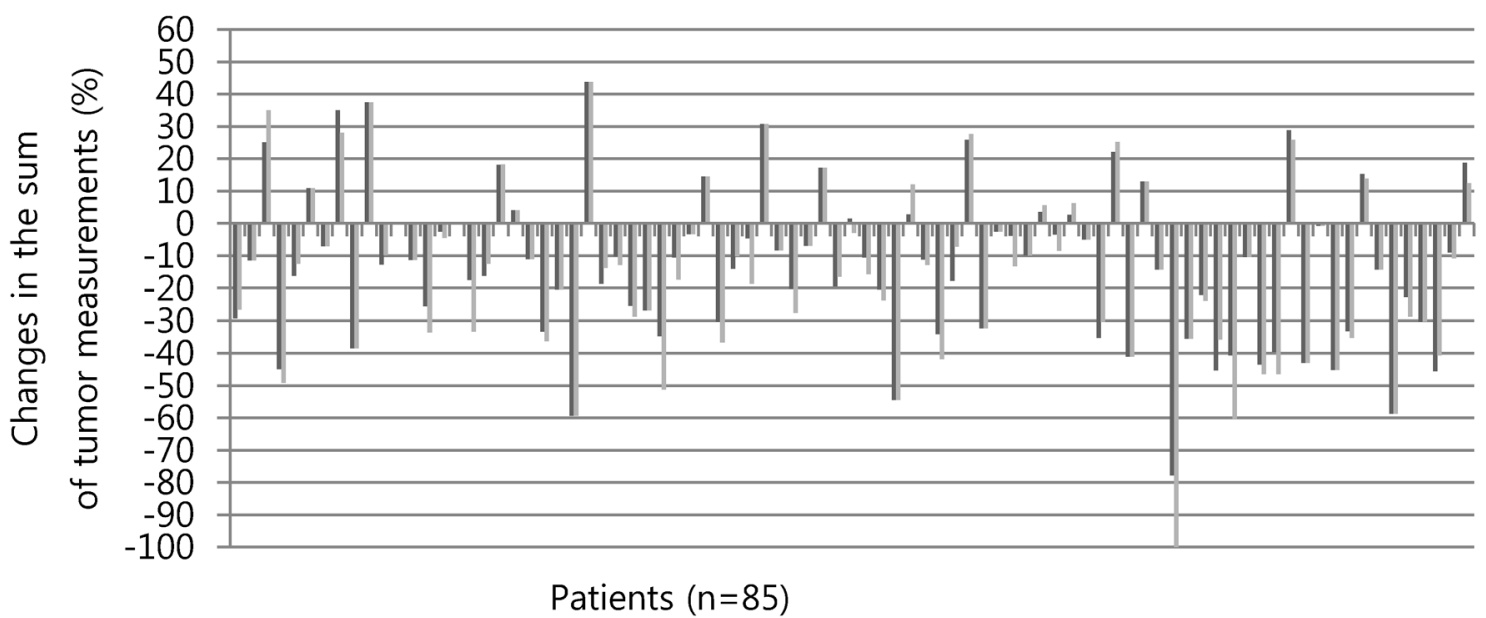

Figure 1. Percentage changes in the sum of tumor measurements according to the RECIST 1.0 versus RECIST 1.1. Three patients who no longer had target lesions according to the RECIST 1.1 were not shown here. * The last patients who had PD according to the RECIST 1.0 was re-categorized as SD according to the RECIST 1.1 because a new $L N \leq 10 \mathrm{~mm}$ along short axis did not meet the pathologic $L N$ criteria. 
The decrease of target lesions by adopting the RECIST 1.1 was mainly caused by the change of the LN evaluation criteria. The new LN criteria of the RECIST 1.1 affected the number of target lesions in 39 patients $(44.3 \%)$. Out of 88 patients who had at least one target lesion according to the RECIST 1.0 at baseline, 3 (3.4\%) no longer had target lesions because of the pathologic LN criteria of the RECIST 1.1. As a result, the proportion of patients with at least one target lesion at baseline was slightly reduced from $92.7 \%$ by the RECIST 1.0 to $90.2 \%$ by the RECIST 1.1. Although there was no statistical significance, these three patients would have been excluded from clinical trials if studies using the RECIST 1.1 as response criteria had been planned. Fuse and colleagues reported that the proportion of patients with target lesions was significantly decreased from $67 \%$ to $53 \%$ by the new $\mathrm{LN}$ criteria in patients with advanced GC [13]. Jang and colleagues also observed the significant decline of patients with at least one measureable lesion in metastatic CRC (from $86.1 \%$ by the RECIST 1.0 to $77.8 \%$ by the RECIST 1.1) [15]. These findings indicate that the RECIST 1.1 may alter the eligibility of patients for clinical trials with ORR or time to progression as primary endpoints. The change in the maximum number of target lesions (from 10 to 5 in total, and from 5 to 2 per organ) affected 13 patients. They had more than 3 target lesions in one organ, such as the lungs, LNs, or liver, but only two largest lesions counted as target lesions according to the RECIST 1.1.

In the current study, patients received cytotoxic chemotherapy, not target agents, as first-line treatment for advanced NSCLC. Although this study had a relatively small number of patients, tumor responses showed almost perfect agreement between the two RECIST versions $(\mathrm{k}=0.912)$. As a result, the ORRs of first-line chemotherapy were not significantly different between the two criteria $(27.1 \%$ by the RECIST 1.0 and $29.4 \%$ by the RECIST 1.1 ). Our results are also consistent with the prior reports conducted in patients with metastatic GC or CRC treated with cytotoxic chemotherapy [13-15]. Jang and colleagues reviewed 58 metastatic CRC patients who received first-line chemotherapy in clinical practice [15]. Although the number of target lesions according to the RECIST 1.1 decreased in $82.7 \%$ of patients $(48 / 58), 94.2 \%$ of patients showed the same classification of the tumor response between the two criteria. In the study of advanced GC by Fuse and colleagues, the ORR was not significant different between the two criteria (52\% according to the RECIST 1.0 and 55\% according to the RECIST 1.1).

When the RECIST 1.1 was revised, patients treated with target agents were not included in the data warehouse [9]. Target agents tend to induce ne- crosis and cystic change in solid tumors without necessarily producing tumor shrinkage [20]. However, the RECIST 1.1 has also shown high concordance in patients with NSCLC or thyroid cancer treated with a target agent such as EGFR-TKIs [16-19, 21]. These findings suggest that the RECIST 1.1 can be applied to patients with NSCLC regardless of the type of treatment. The new LN criteria of the RECIST 1.1 were also a major cause of the reclassification of tumor response in other study of NSCLC patients treated with EGFR-TKIs [17]. In our study, four patients (4.5\%) showed disagreement of the tumor responses between the two criteria and all of these disagreements were due to the change in the LN measurement criteria.

In conclusion, the RECIST 1.1 provided a high level of concordance with the RECIST 1.0 in the assessment of tumor response in advanced NSCLC patients treated with cytotoxic chemotherapy. The new LN criteria of the RECIST 1.1 were a major cause of the reduction of target lesions and reclassification of tumor response. The RECIST 1.1 may also alter the eligibility of patients for clinical trials with ORR or time to progression as primary endpoints.

\section{Conflict of Interest}

Authors do not have any conflict of interest.

\section{References}

1. Miller AB, Hoogstraten B, Staquet M, Winkler A. Reporting results of cancer treatment. Cancer. 1981;47:207-14

2. Therasse P. Measuring the clinical response. What does it mean? Eur J Cancer. 2002;38:1817-23.

3. Choi JH, Ahn MJ, Rhim HC, Kim JW, Lee GH, Lee YY, et al. Comparison of WHO and RECIST criteria for response in metastatic colorectal cancer. Cancer Res Treat. 2005;37:290-3.

4. Park JO, Lee SI, Song SY, Kim K, Kim WS, Jung CW, et al. Measuring response in solid tumor: comparison of RECIST and WHO criteria. Jpn J Clin Oncol. 2003;33: 533-7.

5. Therasse P, Arbuck SG, Eisenhauer EA, Wanders J, Kaplan RS, Rubinstein L, et al. New guidelines to evaluate the response to treatment in solid tumors. European Organization for Research and Treatment of Cancer, National Cancer Institute of the United States, National Cancer Institute of Canada. J Natl Cancer Inst. 2000;92:205-16.

6. Sargent DJ, Rubinstein L, Schwartz L, Dancey JE, Gatsonis C, Dodd LE, et al. Validation of novel imaging methodologies for use as cancer clinical trial endpoints. Eur J Cancer. 2009;45:290-9.

7. Moskowitz CS, Jia X, Schwartz LH, Gonen M. A simulation study to evaluate the impact of the number of lesions measured on response assessment. Eur J Cancer. 2009;45:300-10.

8. Eisenhauer EA, Therasse P, Bogaerts J, Schwartz LH, Sargent D, Ford R, et al. New response evaluation criteria in solid tumours: revised RECIST guideline (version1.1). Eur J Cancer. 2009;45:228-47.

9. Bogaerts J, Ford R, Sargent D, Schwartz LH, Rubinstein L, Lacombe D, et al. RECIST Working Party: Individual patient data analysis to assess modifications to the RECIST criteria. Eur J Cancer. 2009;45:248-60.

10. Schwartz LH, Bogaerts J, Ford R, Shankar L, Therasse P, Gwyther S, et al. Evaluation of lymph nodes with RECIST 1.1. Eur J Cancer. 2009;45:261-7.

11. Dancey JE, Dodd LE, Ford R, Kaplan R, Mooney M, Rubinstein L, et al. Recommendations for the assessment of progression in randomized cancer treatment trials. Eur J Cancer. 2009;45:281-9.

12. van Persijn van Meerten EL, Gelderblom H, Bloem JL. RECIST revised: implications for the radiologist. A review article on the modified RECIST guideline. Eur Radiol. 2010;20:1456-67.

13. Fuse N, Nagahisa-Oku E, Doi T, Sasaki T, Nomura S, Kojima T, et al. Effect of RECIST revision on classification of target lesions and overall response in advanced gastric cancer patients. Gastric Cancer. 2013;16:324-8. 
14. Jang GS, Kim MJ, Ha HI, Kim JH, Kim HS, Ju SB, et al. Comparison of RECIST version 1.0 and 1.1 in assessment of tumor response by computed tomography in advanced gastric cancer. Chin J Cancer Res. 2013;25:689-94.

15. Jang HJ, Kim BC, Kim HS, Kim JH, Song HH, Kim JB, et al. Comparison of RECIST 1.0 and RECIST 1.1 on computed tomography in patients with metastatic colorectal cancer. Oncology. 2014;86:117-21.

16. Ruan M, Shen Y, Chen L, Li M. RECIST 1.1 and serum thyroglobulin measurements in the evaluation of responses to sorafenib in patients with radioactive iodine-refractory differentiated thyroid carcinoma. Oncol Lett. 2013;6:480-6.

17. Sun JM, Ahn MJ, Park MJ, Yi JH, Kim TS, Chung MJ, et al. Accuracy of RECIST 1.1 for non-small cell lung cancer treated with EGFR tyrosine kinase inhibitors. Lung Cancer. 2010;69:105-9.

18. Nishino M, Cardarella S, Jackman DM, Ramaiya NH, Rabin MS, Hatabu H, et al. RECIST 1.1 in NSCLC patients with EGFR mutations treated with EGFR tyrosine kinase inhibitors: comparison with RECIST 1.0. AJR Am J Roentgenol. 2013;201:64-71.

19. Nishino M, Jackman DM, Hatabu H, Yeap BY, Cioffredi LA, Yap JT, et al. New Response Evaluation Criteria in Solid Tumors (RECIST) guidelines for advanced non-small cell lung cancer: comparison with original RECIST and impact on assessment of tumor response to target therapy. AJR Am J Roentgenol. 2010;195:221-8.

20. Shankar LK, Van den Abbeele A, Yap J, Benjamin R, Scheutze S, Fitzgerald TJ. Considerations for the use of imaging tools for phase II treatment trials in oncology. Clin Cancer Res. 2009;15:1891-7.

21. Kim JH, Min SJ, Jang HJ, Cho JW, Kim SH, Kim HS. Comparison of RECIST 1.0 and RECIST 1.1 in patients with metastatic cancer: a pooled analysis. J Cancer. 2015;6:387-93. 